\title{
Field tuning and rf measurements of the four-vane radio frequency quadrupole with ramped inter-vane voltage
}

\author{
Q. Z. Xing, ${ }^{1,2,3,}$ X. D. Yu, ${ }^{1,2,3}$ Q. K. Guo, ${ }^{1,2,3}$ P. F. Ma, ${ }^{1,2,3}$ Y. Lei, ${ }^{1,2,3}$ S. X. Zheng, ${ }^{1,2,3}$ Y. Li, ${ }^{1,2,3}$ \\ S. Wang, ${ }^{1,2,3}$ K. Liu, ${ }^{1,2,3}$ X. L. Guan, ${ }^{1,2,3}$ X. W. Wang, ${ }^{1,2,3}$ B. C. Wang, ${ }^{4}$ \\ C. Zhao, ${ }^{4}$ and Z. M. Wang ${ }^{4}$ \\ ${ }^{1}$ Key Laboratory of Particle \& Radiation Imaging (Tsinghua University), \\ Ministry of Education, Beijing 100084, China \\ ${ }^{2}$ Laboratory for Advanced Radiation Sources and Application, Tsinghua University, \\ Beijing 100084, China \\ ${ }^{3}$ Department of Engineering Physics, Tsinghua University, Beijing 100084, China \\ ${ }^{4}$ State Key Laboratory of Intense Pulsed Radiation Simulation and Effect \\ (Northwest Institute of Nuclear Technology), Xi'an 710024, China
}

(Received 6 September 2018; published 19 February 2019)

\begin{abstract}
This paper describes the physical design, rf measurement, and tuning result of a $325 \mathrm{MHz}$ four-vane radio frequency quadrupole (RFQ) with a ramped inter-vane voltage. The 3-meter-long RFQ will accelerate the negative hydrogen ion beam from $50 \mathrm{keV}$ to $3 \mathrm{MeV}$ with a peak current of $5 \mathrm{~mA}$, a pulse width of $10-40 \mu \mathrm{s}$, and a maximum repetition rate of $0.5 \mathrm{~Hz}$. During tuning we reveal that the coupling loop of the coaxial coupler adopted for this RFQ enhances the interference between the dipole field distribution and the coupling coefficient. Therefore, an iteration method is adopted to simultaneously achieve the desired field distribution and coupling coefficient. After tuning, the relative error between the measured field distribution and the designed curve for the operating quadrupole mode is within $\pm 2.7 \%$, and the dipole components are about $\pm 1.9 \%$ of the quadrupole mode. The measured coupling coefficient of the rf power coupler equals 1.03 , with the desired value of 1.04 .
\end{abstract}

DOI: 10.1103/PhysRevAccelBeams.22.020102

\section{INTRODUCTION}

A $3 \mathrm{MeV}$ radio frequency quadrupole (RFQ) has been designed [1], manufactured [2] and tuned [3] for the $7 \mathrm{MeV}$ linac injector for the synchrotron of the Xi' an $200 \mathrm{MeV}$ Proton Application Facility (XiPAF). The XiPAF project started construction in 2014 to fulfill the need for experimental simulation of space radiation environments, especially for the investigation of single event effects. The facility is mainly composed of a $7 \mathrm{MeV} \mathrm{H}^{-}$linac injector, a middle energy beam transport line (MEBT), a $200 \mathrm{MeV}$ six-fold synchrotron, two high energy beam transport lines (HEBTs) and two experimental stations. A flux of $10^{5}-10^{8} \mathrm{p} / \mathrm{cm}^{2} / \mathrm{s}$ with a uniformity better than $90 \%$ on a $10 \mathrm{~cm} \times 10 \mathrm{~cm}$ sample is designed for the experimental stations.

The linac injector (Fig. 1) consists of a $50 \mathrm{keV}$ negative hydrogen ion source, a low energy beam transport line

\footnotetext{
*xqz@tsinghua.edu.cn
}

Published by the American Physical Society under the terms of the Creative Commons Attribution 4.0 International license. Further distribution of this work must maintain attribution to the author(s) and the published article's title, journal citation, and DOI.
(LEBT), an RFQ accelerator, and an Alvarez-type drift tube linac (DTL). With a total length of $5198 \mathrm{~mm}$, the RFQ and DTL will consume an rf power of $669 \mathrm{~kW}$.

The RFQ will accelerate the negative hydrogen ion beam from $50 \mathrm{keV}$ to $3 \mathrm{MeV}$ with a peak current of $5 \mathrm{~mA}$, a pulse width of $10-40 \mu \mathrm{s}$, and a maximum repetition rate of $0.5 \mathrm{~Hz}$. The RFQ will be powered by a 4616V4 tetrode amplifier, and the power will be fed into the RFQ cavity through a coaxial power coupler via loop-coupling. Undercuts [4] exist on the two end segments, thus creating a looped circuit of the magnetic field. The dipole-mode stabilizer rods [5] are adopted to keep the frequency separation between the operating quadrupole mode and its neighboring dipole modes as large as possible. The intervane voltage is increased with the longitudinal position, and this benefits the production of a 3-meter-long RFQ; therefore, coupling plates [6] are not needed to enhance the field stability.

Nevertheless, the field distribution is important for the operation of an RFQ. The relatively large error of the field distribution can lead to changes in the synchronous energy, Twiss parameters, and transmission efficiency of the beam at the RFQ exit. The loop coupling which has been adopted for this RFQ will lead to an interaction between the field distribution and the coupling coefficient. Therefore, besides 


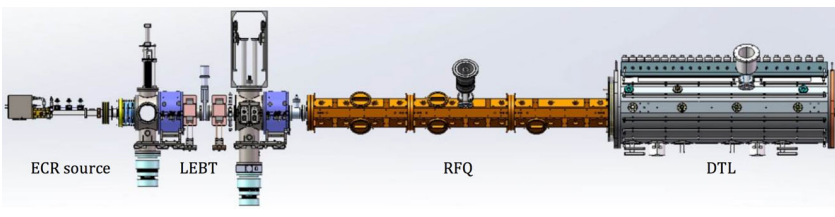

FIG. 1. $7 \mathrm{MeV} \mathrm{H}^{-}$linac injector for the synchrotron of the Xi'an $200 \mathrm{MeV}$ Proton Application Facility.

achieving the desired quadrupole field distribution and a small component of the dipole field, the coupling coefficient must be adjusted to the required value simultaneously during the tuning. Furthermore, adequate length of the dipole-mode stabilizer rods should be verified in the tuning experiment to confirm the large frequency separation between the operating quadrupole mode and the nearest dipole mode.

The iris coupler and coaxial coupler are commonly used as rf power couplers for RFQs. To tune the rf coupling, the diameters of the iris holes have to be adjusted by machining for the iris coupler. The advantage of the iris coupling is that the interaction between the field distribution and coupling coefficient is weak. The tuning of the coupling and the field can be separated. For the LINAC4 RFQ [7] and CPHS RFQ [8], the tuning of the field follows the tuning of the coupling; whereas for the LEDA RFQ [9], the tuning of the field occurs first. For the loop coupling, tuning the coupling is easily done by only rotating the coaxial coupler and adjusting the azimuthal angle of the loop; however a relatively stronger interaction is present between the dipole field distribution and coupling coefficient. Loop coupling is adopted for the $750 \mathrm{MHz}$ RFQ at CERN, with designed and measured coupling coefficient of 1.18 and 1.03 separately [10]; however, the detailed tuning process of the rf coupling is not presented.

In this paper, an iteration method is adopted to simultaneously achieve the desired field distribution and coupling coefficient for the XiPAF RFQ with a coaxial coupler. This paper is organized as follows: Section II presents the dynamics design and rf structure design of the XiPAF RFQ. Section III describes the tuning method. Section IV presents the experimental setup, tuning process and final result. Finally, the conclusions are summarized in Sec. V.

\section{PHYSICAL DESIGN}

\section{A. Beam dynamics design}

The four-vane RFQ is designed to accelerate a $50 \mathrm{keV}$ $\mathrm{H}^{-}$beam to $3 \mathrm{MeV}$, and deliver it to the downstream DTL with the peak current of $5 \mathrm{~mA}$. The main design parameters of the XiPAF RFQ are listed in Table I. The design result of the various parameters of the RFQ is shown in Fig. 2, in which $B$ is the focusing strength, $X$ is the focusing parameter, $A$ is the acceleration parameter, $W$ is the synchronous energy, $\Phi_{\mathrm{s}}$ is the synchronous phase, $m$ is the modulation factor, $r_{0}$ is the mean bore radius, and $a$ is
TABLE I. Main design parameters of XiPAF RFQ.

\begin{tabular}{llc}
\hline \hline Parameters & Value & Unit \\
\hline Particle & $\mathrm{H}^{-}$ & \\
Input energy & 50 & $\mathrm{keV}$ \\
Output energy & 3 & $\mathrm{MeV}$ \\
Frequency & 325 & $\mathrm{MHz}$ \\
Peak current intensity & 5 & $\mathrm{~mA}$ \\
Maximum reputation rate & 0.5 & $\mathrm{~Hz}$ \\
Beam pulse length & $10-40$ & $\mu \mathrm{s}$ \\
$Q_{0}(0.8 *$ SUPERFISH) & 8600 & \\
Peak wall power $\left(Q_{0}=8600\right)$ & 388 & $\mathrm{~kW}$ \\
\hline \hline
\end{tabular}

the minimum bore radius. The inter-vane voltage is set to increase with the longitudinal position, while limiting the peak surface electric field to 1.8 Kilpatrick. The increased voltage can substantially enhance the accelerating field, thereby shortening the RFQ, while maintaining a relatively large aperture.

\section{B. rf structure design}

The XiPAF RFQ is mechanically separated into three segments to facilitate machining and brazing. Each of the three segments has a length about 1 meter. Moreover no coupling plate will be adopted between the adjacent segments. The structure drawing of the XiPAF RFQ is shown in Fig. 3.

\section{Vane structure}

The cavity cross section and vane-tip geometry are tailored as a function of longitudinal position to keep the desired local frequency. One cross section of the RFQ and the field calculated by using the SUPERFISH code [11] are shown in Fig. 4. The frequency of the operating $\mathrm{TE}_{210}$ mode is designed to be $323.5 \mathrm{MHz}$ with all the tuners flush

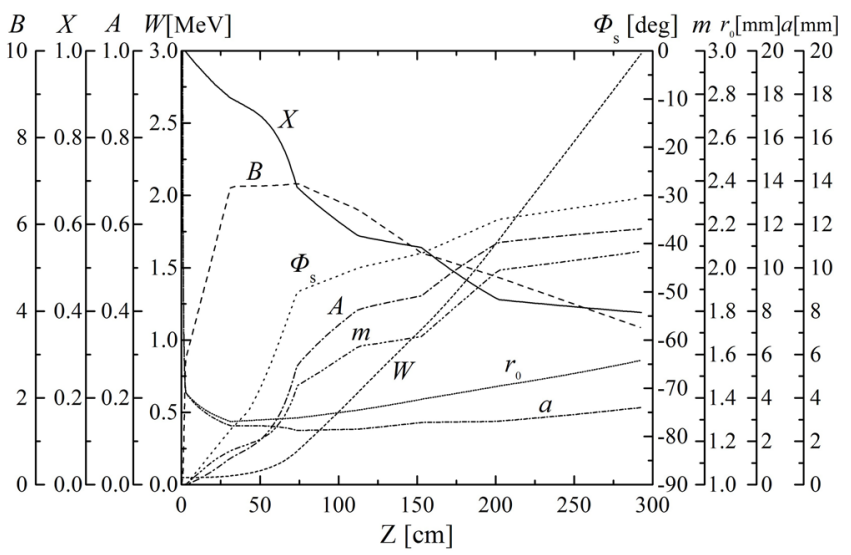

FIG. 2. Various parameters of the XiPAF RFQ versus longitudinal position: $B$ is the focusing strength, $X$ is the focusing parameter, $A$ is the acceleration parameter, $W$ is the synchronous energy, $\Phi_{\mathrm{s}}$ is the synchronous phase, $m$ is the modulation factor, $r_{0}$ is the mean bore radius, and $a$ is the minimum bore radius. 


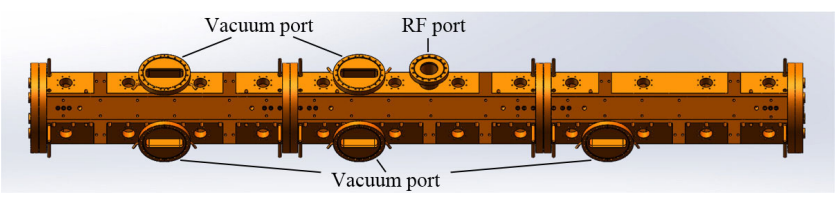

FIG. 3. Structure drawing of the XiPAF RFQ.

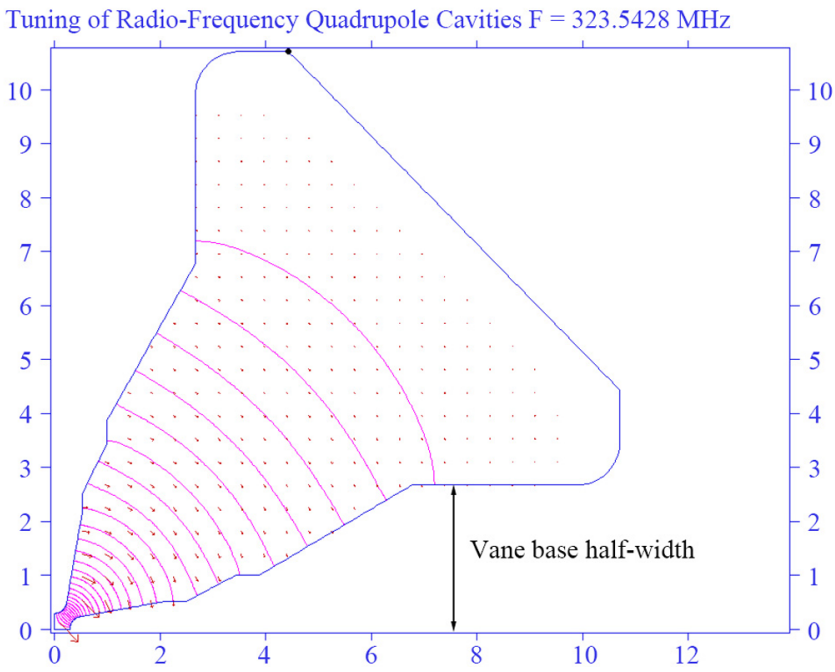

FIG. 4. Cross section of the RFQ at $z=33.2 \mathrm{~cm}$ with the field calculated by the SUPERFISH code.

with the interior wall of the RFQ. The half-width of the vane base and inter-vane voltage versus the longitudinal position are presented in Fig. 5.

\section{Undercut and dipole-mode stabilizer rod}

Undercuts on each end of the RFQ help achieve a ramped field distribution close to the desired one. An

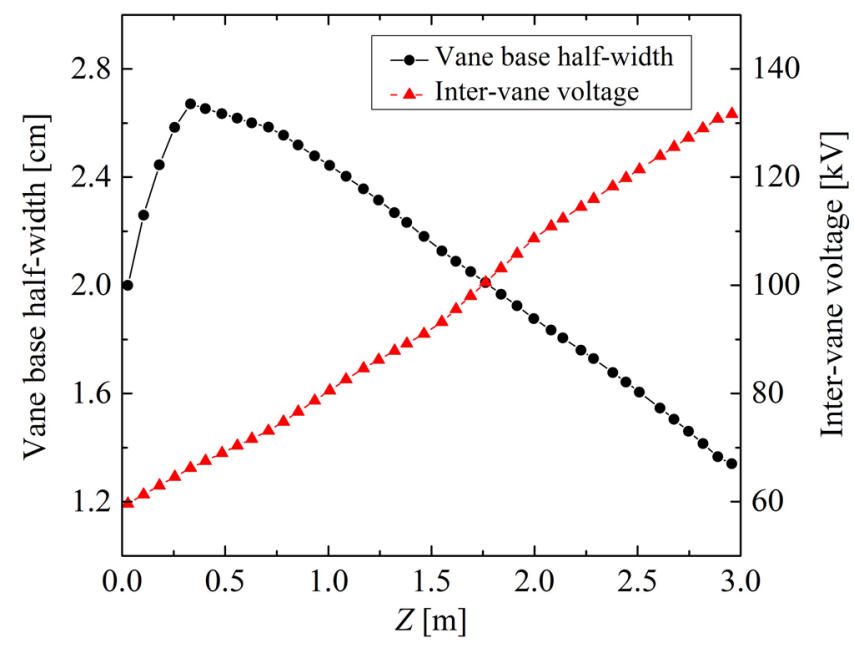

FIG. 5. Half-width of the vane base and inter-vane voltage versus the longitudinal position.

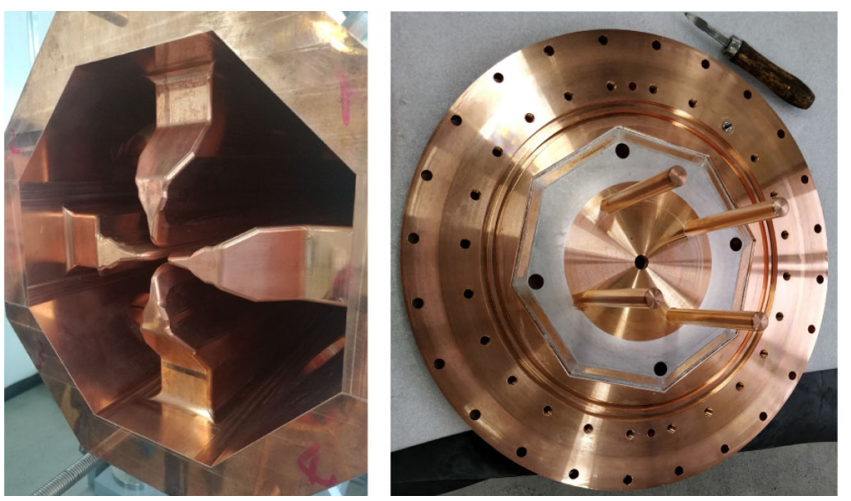

FIG. 6. Undercuts (left) and dipole-mode stabilizer rods (right) of the XiPAF RFQ.

adequate design will facilitate the tuning of the field. Four dipole-mode stabilizer rods are mounted on each of the two end flanges. The rod positions are determined by the SUPERFISH code to keep the frequency of the $\mathrm{TE}_{210}$ mode unvaried. The frequency interval between $\mathrm{TE}_{210}$ and its nearest neighboring $\mathrm{TE}_{111}$ modes is $5.6 \mathrm{MHz}$, with the optimized length of all the rods being $14.7 \mathrm{~cm}$. The final rod length will be determined during the tuning. The undercuts and dipole-mode stabilizer rods of the XiPAF RFQ are shown in Fig. 6.

\section{Tuner}

A total of 48 tuners with the diameter of $48 \mathrm{~mm}$ are distributed in the four quadrants of the RFQ cavity. Three magnetic feedthroughs used as field probes are separately brazed in three tuners to monitor the field during operation [12]. Structure drawings of one tuner with one probe inside and tuners inside the RFQ cavity are shown in Fig. 7.

\section{Power coupler}

The RFQ is powered by one $4616 \mathrm{~V} 4$ tetrode amplifier, and the power is fed into the RFQ through one coaxial power coupler via loop-coupling [13]. The coupling
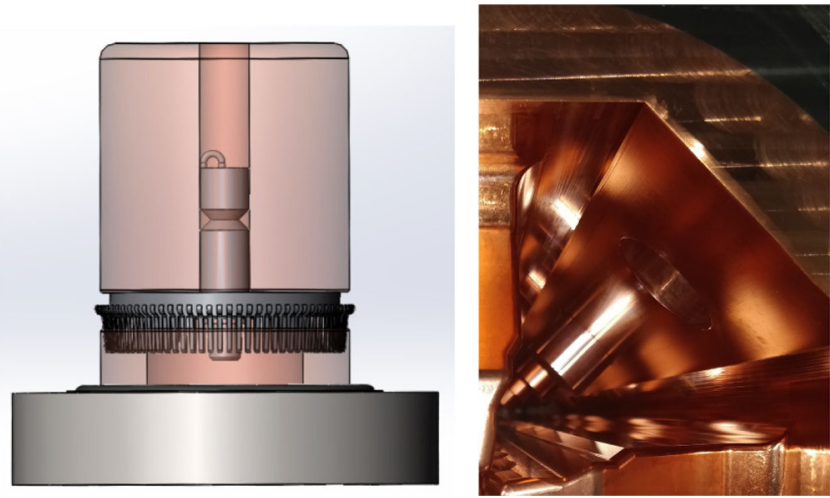

FIG. 7. Structure drawings of one tuner with one probe inside (left) and tuners inside the RFQ cavity (right). 


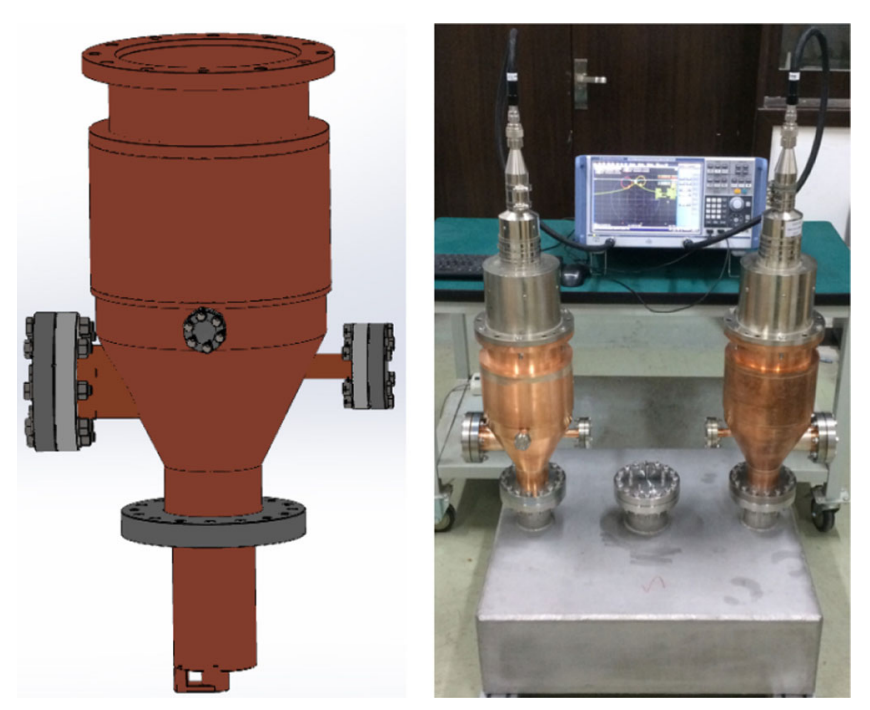

FIG. 8. Structure drawing of the power coupler (left) and two power couplers connecting with one power-conditioning cavity (right).

coefficient needs to be determined by the measured intrinsic quality factor of the RFQ cavity. Figure 8 shows the structure drawing of the power coupler, and two manufactured power couplers are mounted on one power-conditioning cavity. One of the two couplers is a spare.

\section{TUNING METHOD}

For the XiPAF RFQ, the main difficulties include achieving the desired quadrupole field distribution and a small component of the dipole field, while simultaneously achieving the required coupling coefficient. In this section the algorithm for field tuning is discussed first, and then the tuning of the rf coupling is considered and described.

\section{A. Tuning algorithm}

As in a four-vane RFQ, the magnetic fields of the quadrupole and dipoles of a four-vane RFQ are displayed in Fig. 9 [14].

The magnetic fields of the quadrupole and dipole modes can be obtained by decomposing the field distributions in four quadrants:

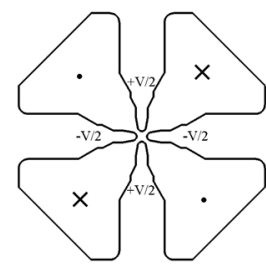

Quadrupole

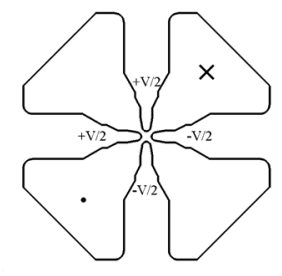

$D_{13}$

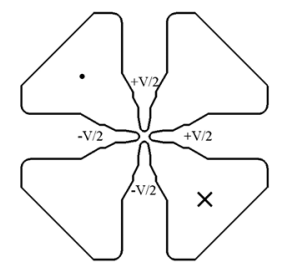

$D_{24}$
FIG. 9. Diagrams of the magnetic field for the quadrupole and dipoles of a four-vane RFQ.

$$
\begin{aligned}
Q & =\left(H_{1}+H_{2}+H_{3}+H_{4}\right) / 4 \\
D_{13} & =\left(H_{1}-H_{3}\right) / 2 \\
D_{24} & =\left(H_{2}-H_{4}\right) / 2
\end{aligned}
$$

where $Q$ is the amplitude of the quadrupole mode, $D_{13}$ and $D_{24}$ are the amplitudes of the dipole modes in Fig. 9, and $H_{1}, H_{2}, H_{3}$, and $H_{4}$ are the amplitudes of the magnetic field in the four quadrants.

In the RFQ, the field distributions of the dipole and quadrupole modes are respectively a set of standard orthogonal and complete bases. By connecting the field distribution quadrant by quadrant to form a field $H_{0}$, the field can be described as the superposition of the first bases [15]:

$$
H_{0}(z, q)=\sum_{n=1}^{3 N} \kappa(n) X(z, q, n)
$$

where $X(z, q, n)$ is a vector recorded as $(Q(z, q, 1), \ldots$ $Q(z, q, N), D_{13}(z, q, N+1), \ldots D_{13}(z, q, 2 N), D_{24}(z, q$, $\left.2 N+1), \ldots D_{24}(z, q, 3 N)\right)^{\mathrm{T}}$, with the meaning of the corresponding $n$ th-mode field at the longitudinal position of $z$ in the qth quadrant; $\kappa(n)$ is the coefficients of the $n$th mode; and $N$ is infinite theoretically, but the sum can be truncated to $N=7$ in our case.

Field tuning aims to obtain the desired coefficients $\kappa_{0}(n)$, which are derived from the required field distribution, by adjusting the insertion depths of the tuners. Tuning is an iterative process, which can be performed with the THURT code developed at the Tsinghua University [15]. Suppose that the acquired coefficients are $\kappa_{i}(n)$ after the $i$ th iteration, we must introduce a coefficient correction of $\delta \kappa_{i+1}=\kappa_{0}-\kappa_{i}$ in the next iteration. According to the standard perturbation theory [16], a relationship exists between $\delta \kappa$ and the frequency perturbation $\delta f$ due to the alteration of insertion depths of the tuners:

$$
\delta \kappa=\mathbf{M} \cdot \delta f
$$

where $\delta \kappa \in R^{3 N}$ denotes the coefficient correction of the $3 N$ modes, $\delta f \in R^{K}$ refers to the $K$ values of the frequency perturbation for $K$ tuners, and $\mathbf{M} \in R^{3 N \times K}$ corresponds to the mapping matrix, with the $(n j)^{\text {th }}$ element of

$$
M_{n j}=\frac{2 f_{0} X(z(j), q(j), n)}{f_{0}^{2}-f_{n}^{2}}
$$

where $z(j)$ and $q(j)$ refer to the longitudinal position and quadrant of $j$ th tuner, respectively. $f_{n}$ and $f_{0}$ denote the frequency and cut-off frequency of the $n$ th-mode base, respectively. From Eq. (3) we can obtain the frequency perturbation needed for the tuners: 


$$
\delta f=\mathbf{M}^{-1} \cdot \delta \kappa
$$

With the relationship between the frequency shift and insertion depths of the tuners, the program can provide the insertion depths of all the tuners for the $(i+1)$ th iteration.

To simultaneously tune the field distribution and resonant frequency, Eq. (3) is replaced with the following:

$$
\left[\begin{array}{c}
\delta_{1} \\
\vdots \\
\delta_{3 N} \\
f-f_{i}
\end{array}\right]=\left[\begin{array}{cccc}
M_{11} & M_{12} & \cdots & M_{1, K} \\
\vdots & \vdots & \ddots & \vdots \\
M_{3 N, 1} & M_{3 N, 2} & \cdots & M_{3 N, K} \\
1 & 1 & \cdots & 1
\end{array}\right]\left[\begin{array}{c}
\delta f_{1} \\
\vdots \\
\delta f_{K}
\end{array}\right]
$$

where $f$ denotes the required resonant frequency, and $f_{i}$ is the measured resonant frequency after the $i$ th iteration.

\section{B. Influence of the coupling loop}

We now consider the effect of rf coupling loop. According to the equivalent circuit model of coupler [17], a coupling loop can be considered as a transformer. When the coupling loop is rotated, the coupling coefficient will change, also causing changes in the capacitance between the coupling loop and vane, and the equivalent impedance of the coaxial at the input end of the transformer. Comparing with the iris coupling of a tapered ridgeloaded waveguide, the existence of capacitance between the coupling loop and vane will lead to a stronger interaction between the dipole field distribution and coupling coefficient. Figure 10 shows the measured relationship between the coupling coefficient $\beta$, resonance frequency $f$, and angle $\theta . \theta$ is defined as the angle between the loop axis and RFQ axis. The resonant frequency changes by about $4 \mathrm{kHz}$ when the coupling coefficient changes from 0.26 to 1.0.

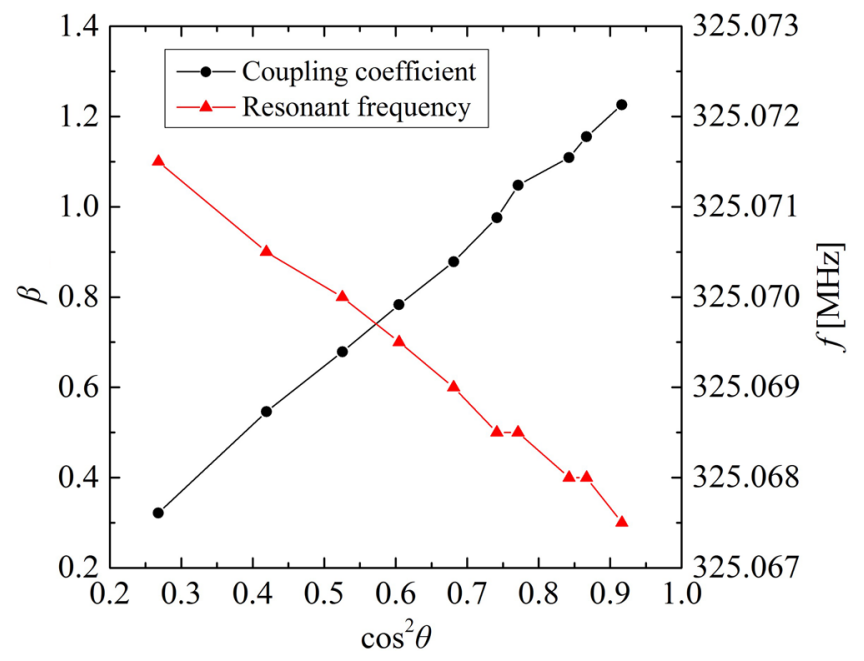

FIG. 10. Measured relationship between the coupling coefficient $\beta$, resonant frequency $f$ and angle $\theta$.

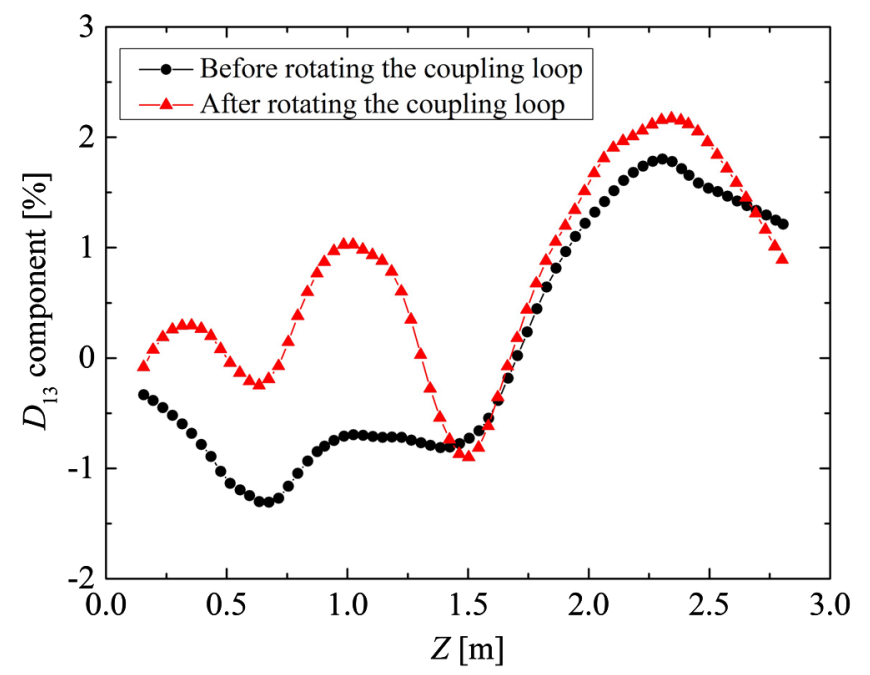

FIG. 11. $D_{13}$ field component before and after rotating the coupling loop by $5.1^{\circ}$.

Figure 11 shows the $D_{13}$ field component measured before and after rotating the coupling loop by $5.1^{\circ}(\theta$ changes from $34.8^{\circ}$ to $39.9^{\circ}$ ); the corresponding coupling coefficient changes from 0.91 to 0.80 . The change in the $D_{13}$ field component can reach $\sim 1.5 \%$ when rotating the coupling loop by $5.1^{\circ}$, which is non-negligible.

\section{Influence of the ramped voltage}

For a ramped-voltage RFQ, the field increases from the low-energy end to high-energy end. As a result, tuners in different positions exhibit different tuning capabilities. Figure 12 shows the frequency perturbation caused by four tuners nearest to the input flange, and four tuners nearest to the output flange. The perturbation of the voltage

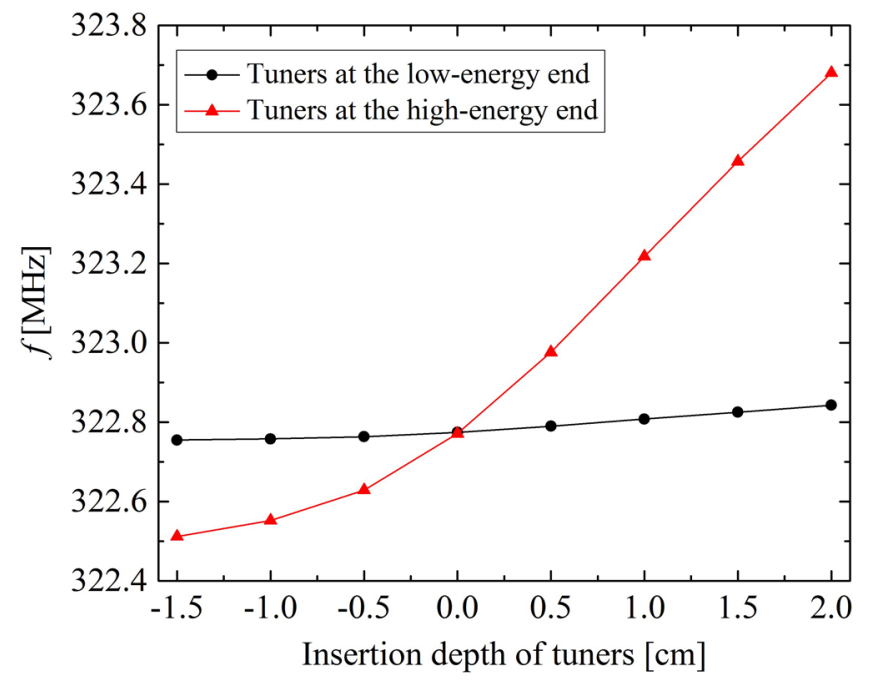

FIG. 12. Relationship between the resonant frequency and insertion depth of four tuners nearest to the input flange, and four tuners nearest to the output flange of the RFQ. 


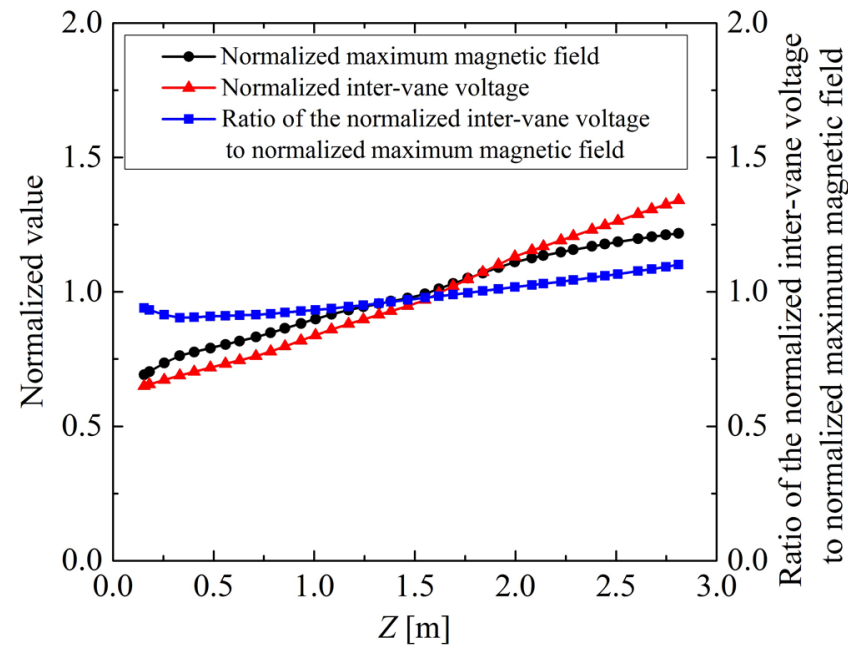

FIG. 13. Designed normalized maximum magnetic field, designed normalized inter-vane voltage distribution, and the ratio between the two variables for the XiPAF RFQ. Both the magnetic field and voltage are normalized by the corresponding RMS (root-mean-square) values over the longitudinal position $Z$.

distribution is proportional to the frequency perturbation [14]. Therefore the tuning capability of tuners at the highenergy end reaches more than ten time that at the lowenergy end. Both the tuning capability of different tuners and coupling coefficient will be affected with the change in the slope of field distribution after each round of tuning. Therefore, the tuning capability of tuners at different positions must be calculated during the tuning process. In each iteration, the weights of all tuners are recalculated by the tuning program from the measured field distribution.

The perturbation theory of tuners is mainly used in RFQ tuning. The conclusion reached by this theory is based on the inter-vane voltage. For constant voltage RFQ, the maximum magnetic field is proportional to inter-vane voltage, and the conclusion of perturbation theory can be directly applied to the maximum magnetic field. However, this proportional relationship no longer holds for the ramped-voltage RFQ. Figure 13 shows the designed normalized maximum magnetic field, designed normalized inter-vane voltage distribution, and the ratio between the two variables for the XiPAF RFQ. The maximum magnetic field near the wall is obtained after the rf structure of the vanes is designed by the RFQfish code. The ratio is no longer constant but close to 1 . Therefore, the maximum magnetic field can still be used instead of the voltage in the perturbation theory. The following tuning process verifies that the approximation is reasonable to obtain the required field.

\section{Tuning strategy}

Figure 14 presents the iteration process of field tuning of the XiPAF RFQ. With this process, both field distribution and coupling coefficient can be tuned simultaneously.

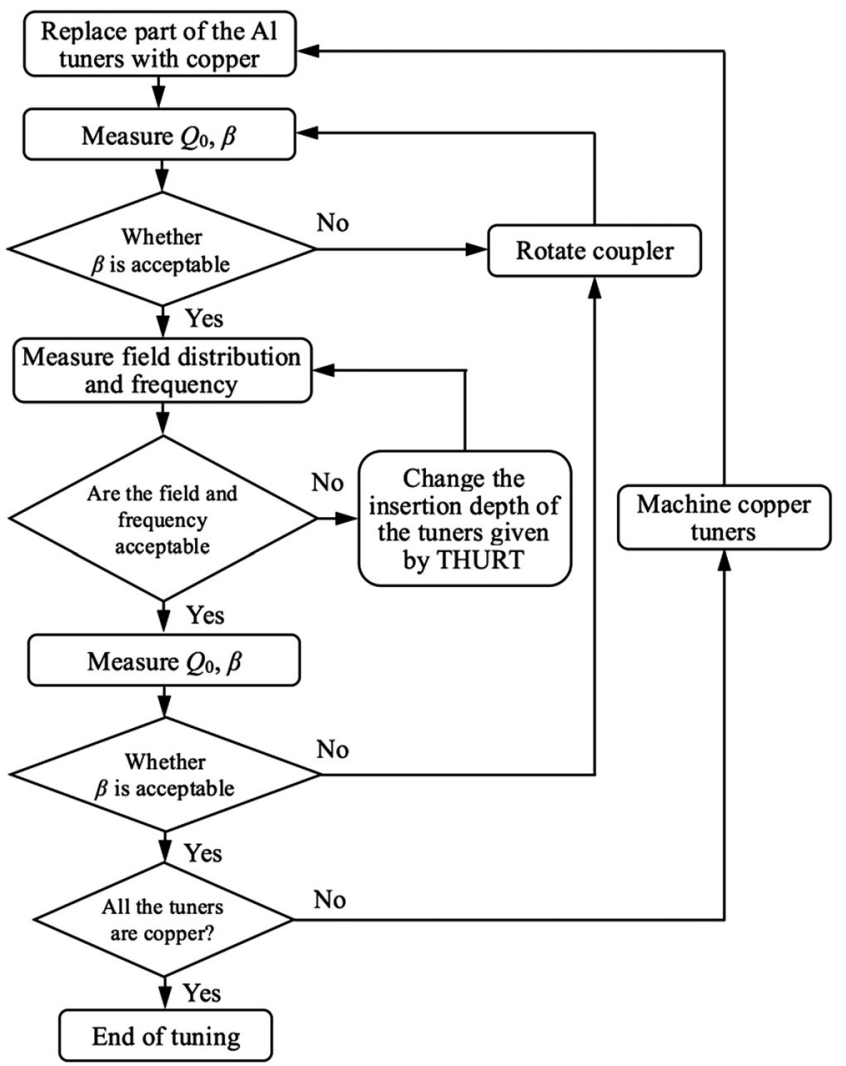

FIG. 14. Flow chart of the field tuning of the XiPAF RFQ.

\section{FIELD TUNING AND RF MEASUREMENTS}

\section{A. Experimental setup}

The machining, brazing, and assembly of the XiPAF RFQ have been completed successfully. After assembly of the three segments, the minimum vane-tip gaps at the position of the tuners and vacuum ports are measured by pin gauges (Fig. 15). Most of the points sampled are within $\pm 50 \mu \mathrm{m}$ of the designed values. The four sets of points represent the gap errors between the measured and designed values for the four quadrants.

Figure 16 shows the assembled XiPAF RFQ and beadpull system at one end of the RFQ for tuning. The magnetic
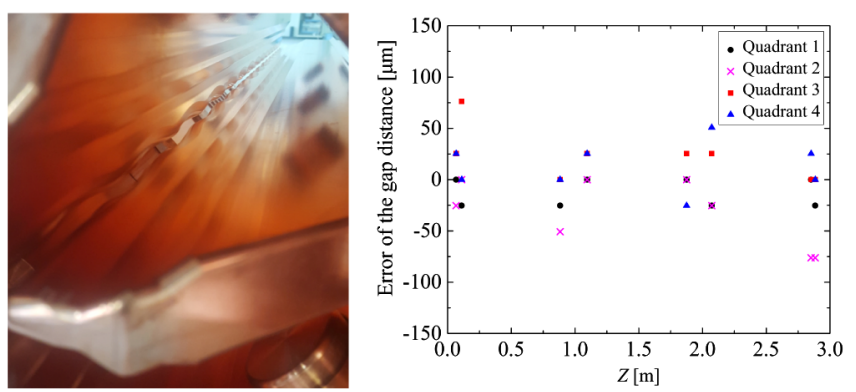

FIG. 15. Vane-tip gaps (left) and error of the gap distance between two adjacent vane tips in four quadrants (right) for the XiPAF RFQ. 

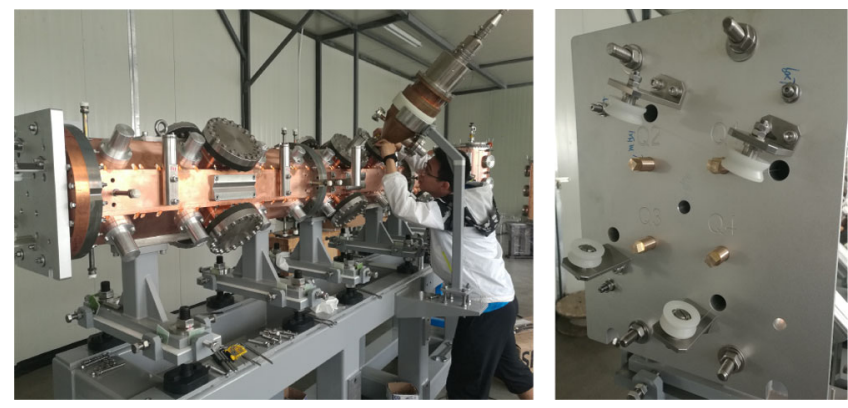

FIG. 16. Assembled XiPAF RFQ (left) and the bead-pull system (right) at one end of the RFQ for tuning.

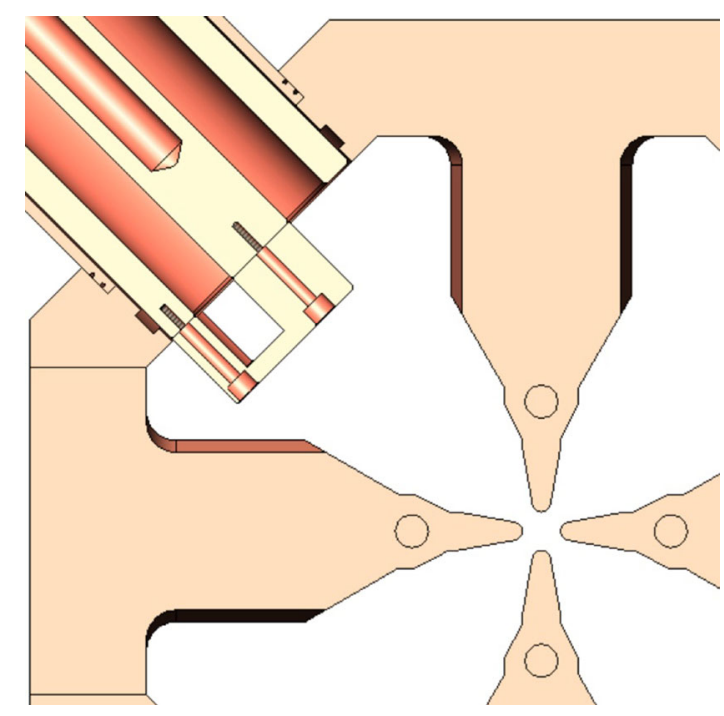

FIG. 17. A cross section of the RFQ at the position of the power coupler.

field distribution near the outer wall is obtained by the bead-pull method. One port of the vector network analyzer is connected to the power coupler, and the other port is connected to a field probe. A hollow metal bead with a diameter of $11.5 \mathrm{~mm}$ is used for perturbation. Field distribution is obtained by measuring the phase change of signal S12.

To improve the signal-to-noise ratio, the phase of signal $\mathrm{S} 12$ is used for field distribution measurement. The measured magnetic field is proportional to the square root of the phase change, $H_{0} \propto \sqrt{\delta \phi}$, with the requirement that the phase of S12 shall be linear with the frequency near the resonant frequency. The linear relationship between the S12 phase and frequency is checked before field measurement. A good linearity is observed in the phase range of $-47^{\circ}$ to $-60^{\circ}$ near the center frequency. From the results of the phase shift with the bead position, the phase ranges from $-46^{\circ}$ to $-54^{\circ}$. The disturbance slightly exceeds the linear region near the first tuner at the high energy end. However, the data near the tuner are not used in the tuning program. Therefore, the method for such measurement is reasonable.

The coaxial power coupler is mounted on the RFQ cavity as shown in Fig. 17. The insertion depth of the loop is $25 \mathrm{~mm}$ and the loop area is $2.3 \mathrm{~cm}^{2}$. The rf coupling can be tuned by rotating the coupler, while the azimuthal angle of the loop is changed simultaneously.

\section{B. Tuning and rf measurements}

The tuning experiment has been carried out starting from 48 aluminum tuners on the XiPAF RFQ. First the lengths of the dipole-mode stabilizer rods are determined. The frequency separation between the operating mode and nearest dipole modes can be adjusted by changing the rod length. Figure 18 shows the measured frequency interval between the operating mode and the nearest dipole modes as function of the rod length. The rod length is set to $15 \mathrm{~cm}$, with a diameter of $1.5 \mathrm{~cm}$. The operating mode is separated from the nearest dipole mode by about 5.2 MHz.

After determining the lengths of the dipole-mode stabilizer rods, the tuning of the field and the rf coupling are performed based on the strategy introduced in Sec. III D. The magnetic field near the outer wall is obtained by the bead-pull method. With the field distribution measured, the tuning program THURT can calculate new insertion depths of the tuners. Figure 19 shows the field distribution in the four quadrants, relative error of the quadrupole field, and dipole component before tuning. The relative error of field distribution of the operating quadrupole mode is within $\pm 25 \%$, and the two dipole modes component are within $\pm 12.8 \%$ of the quadrupole mode.

Tuning is completed after several iterations. The aluminum tuners are replaced by copper ones with a fixed insertion depth step by step. After the field tuning and rf coupling tuning were completed by using the aluminum tuners, about half of the aluminum tuners were replaced by

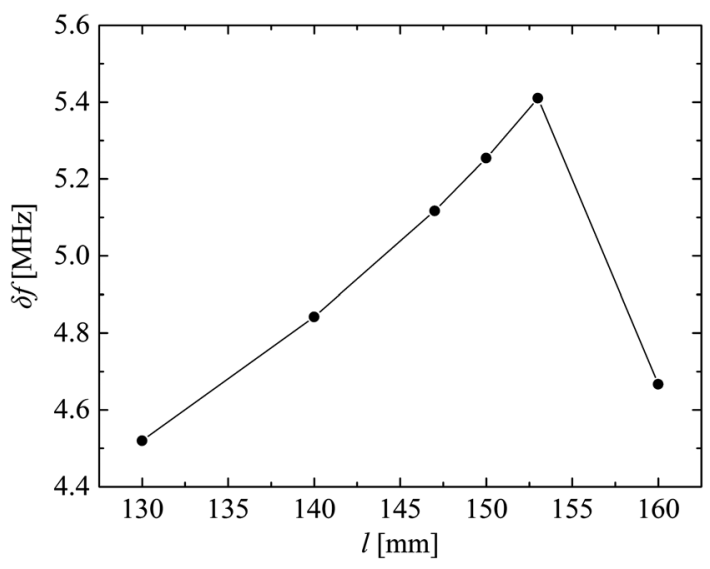

FIG. 18. Measured frequency interval between the operating mode and nearest dipole modes with the rod length. 

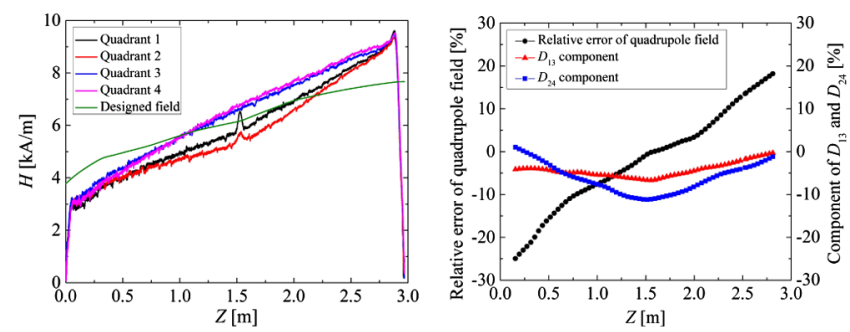

FIG. 19. Field distribution in the four quadrants (left), relative error of the quadrupole field, and dipole component (right) before tuning.
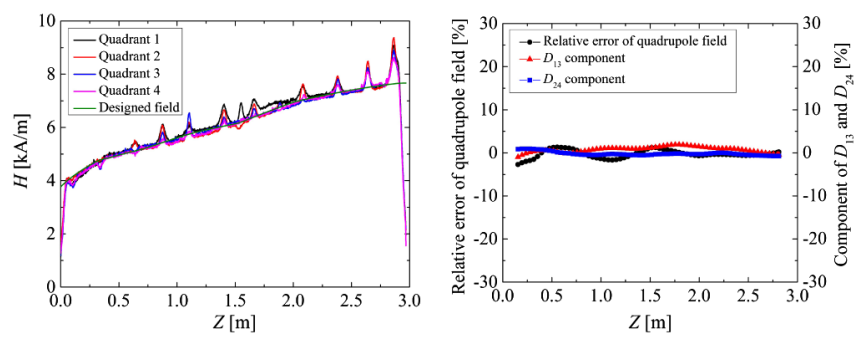

FIG. 20. Field distribution in the four quadrants (left), relative error of the quadrupole field, and dipole component (right) after tuning. $\left(23.7^{\circ} \mathrm{C}\right.$, with nitrogen fed into the cavity).

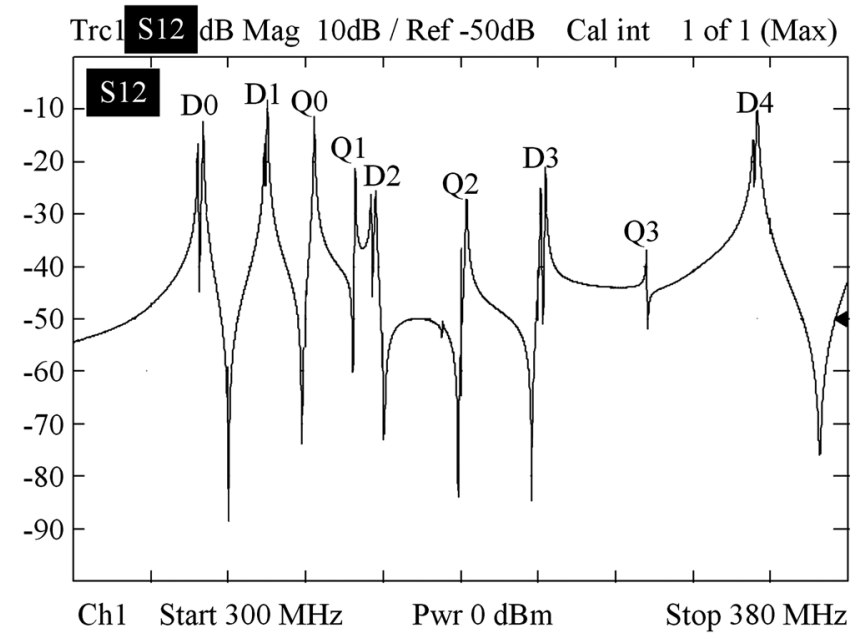

FIG. 21. Frequency spectrum of XiPAF RFQ after tuning. $\left(22^{\circ} \mathrm{C}, 10 \%\right.$ humidity).

copper ones. In the second step half of the rest aluminum tuners were replaced. After three steps, all aluminum tuners were replaced. Figure 20 presents the field distribution in the four quadrants, relative error of the quadrupole field, and dipole component after tuning. The relative error of field distribution of the operating quadrupole mode is within $\pm 2.7 \%$, and the two dipole mode components are about $\pm 1.9 \%$ of the quadrupole mode. Figure 21 shows the frequency spectrum of the XiPAF RFQ after tuning.
The measured intrinsic quality factor $Q_{0}$ is 7300 . With the peak power dissipated on the wall of $457 \mathrm{~kW}$ and the peak beam power of $18 \mathrm{~kW}$, The required coupling coefficient is $\beta=1+P_{\text {beam }} / P_{\text {cavity }}=1.04$. The final measured coupling coefficient of the RF power coupler is 1.03.

\section{CONCLUSIONS}

A $325 \mathrm{MHz}$ four-vane RFQ accelerator with ramped inter-vane voltage has been manufactured for the $7 \mathrm{MeV}$ linac injector of the XiPAF project. The 3-meter-long RFQ will accelerate the negative hydrogen ion beam from $50 \mathrm{keV}$ to $3 \mathrm{MeV}$ with a peak current of $5 \mathrm{~mA}$, a pulse width of 10-40 $\mu \mathrm{s}$, and a maximum repetition rate of $0.5 \mathrm{~Hz}$. The machining, assembly, and rf tuning of the RFQ cavity have been completed successfully.

The RFQ will be powered by one 4616V4 tetrode amplifier, and the power will be fed into the RFQ through one coaxial power coupler by loop-coupling. During tuning, we observe that loop coupling can lead to a non-negligible interaction between the dipole field distribution and the coupler. The change in the dipole field component can reach $\sim 1.5 \%$ when rotating the coupling loop by $5.1^{\circ}$. Therefore, an iteration method is adopted to simultaneously achieve the desired field distribution and coupling coefficient. The iteration process is described.

After tuning, the relative error between the measured field distribution and the designed curve for the operating quadrupole mode is within $\pm 2.7 \%$, and the dipole mode components are about $\pm 1.9 \%$ of the quadrupole mode. The final measured coupling coefficient of the rf power coupler equals 1.03 , with the desired value of 1.04 .

\section{ACKNOWLEDGMENTS}

The authors would like to thank Shanghai Kelin Technology Development CO. LTD for the machining during field tuning.

[1] Q.Z. Xing et al., in Proceedings of IPAC2016 (JACoW, Busan, Korea, 2016), p. 426.

[2] Q.Z. Xing et al., in Proceedings of LINAC2018 (JACoW, Beijing, China, 2018), p. 732.

[3] X. D. Yu, Q. Z. Xing, Q. K. Guo, P. F. Ma, Y. Lei, S. X. Zheng, Y. Li, S. Wang, K. Liu, X. L. Guan, X. W. Wang, B. C. Wang, C. Zhao, and Z. M. Wang, in Proceedings of LINAC2018 (JACoW, Beijing, China, 2018), p. 838.

[4] M. Browman, G. Spalek, and T. Barts, in Proceedings of LINAC1988 (JACoW, Williamsburg, Virginia, USA, 1988), p. 64.

[5] L. M. Young, in Proceedings of LINAC1994 (JACoW, Tsukuba, Japan, 1994), p. 178.

[6] L. M. Young, in Proceedings of PAC1993 (JACoW, New York, USA, 1993), p. 3136. 
[7] O. Piquet, M. Desmons, A. France, Y. Le Noa, J. Novo, and C. Rossi, in Proceedings of IPAC2013 (JACoW, Shanghai, China, 2013), p. 3761.

[8] Q. Z. Xing, L. Du, S. X. Zheng, X. L. Guan, J. Li, J. C. Cai, C. K. Gong, X. W. Wang, C. X. Tang, J. Billen, J. Stovall, and L. Young, Tuning and cold test of a fourvane RFQ with ramped inter-vane voltage for the compact pulsed hadron source, Chin. Phys. Lett. 30, 052901 (2013).

[9] L. M. Young and L. Rybarcyk, in Proceedings of LINAC1998 (JACoW, Chicago, Illinois, USA, 1998), p. 270.

[10] B. Koubek, A. Grudiev, and M. Timmins, rf measurements and tuning of the $750 \mathrm{MHz}$ radio frequency quadrupole, Phys. Rev. Accel. Beams 20, 080102 (2017).

[11] J. Billen and L. Young, Poisson superfish, Los Alamos National Laboratory document Report No. LA-UR-961834, 2005.
[12] https://www.lesker.com/newweb/feedthroughs/ feedthroughs_technicalnotes_instrument.cfm?pgid=0.

[13] Y. Lei, S. X. Zheng, Q. Z. Xing, X. L. Guan, H. Y. Zhang, X. W. Wang, and M. W. Wang, in Proceedings of IPAC2017 (JACoW, Copenhagen, Denmark, 2017), p. 4218.

[14] T. Wangler, Principles of RF Linear Accelerators (JohnWiley \& Sons. Inc., New York, 1998).

[15] L. Du, Q.Z. Xing, S. X. Zheng, X. L. Guan, and C. X. Tang, A fast tuning method for a RFQ accelerator with ramped inter-vane voltage, Nucl. Instrum. Methods Phys. Res., Sect. A 726, 91 (2013).

[16] J. Mathews and R. L. Walker, Mathematical Methods of Physics (WA Benjamin, New York, 1970), Vol. 501.

[17] B. R. Cheo and S. P. Jachim, Dynamic interactions between RF sources and LINAC cavities with beam loading, IEEE Trans. Electron Devices 38, 2264 (1991). 